\title{
Estudo piloto da detecção da mentira pela análise da fluência: é possível?
}

\author{
Pilot study of lie detection by fluency analysis: is it possible? \\ Estudio piloto de la detección de la mentira por el análisis de la fluencia: ¿es posible?
}

\author{
Manoel Moreira de Gois \\ ORCID: https://orcid.org/0000-0003-0055-8672 \\ Universidade Federal de Sergipe, Brasil \\ E-mail: manoelfono@gmail.com \\ Josefa Aparecida Ribeiro Bispo \\ ORCID: https://orcid.org/0000-0001-7553-2122 \\ Universidade Federal de Sergipe, Brasil \\ E-mail: aparecidaribeiro17@gmail.com \\ Mariza de Jesus Santos \\ ORCID: https://orcid.org/0000-0002-8394-9996 \\ Universidade Federal de Sergipe, Brasil \\ E-mail: marizasantostb@hotmail.com \\ Nathália Monteiro Santos \\ ORCID: https://orcid.org/0000-0003-2746-5361 \\ Universidade Federal de Sergipe, Brasil \\ E-mail: nathaliamonteiro.fono@gmail.com \\ José Marcos de Jesus Santos \\ ORCID: https://orcid.org/0000-0001-5122-1469 \\ Universidade de São Paulo, Brasil \\ E-mail: jsmarcos@usp.br \\ Carla Patrícia Hernandez Alves Ribeiro César \\ ORCID: https://orcid.org/0000-0002-9439-9352 \\ Universidade Federal de Sergipe, Brasil \\ E-mail: carlacesar@globo.com
}

\begin{abstract}
Resumo
Objetivo: Analisar os parâmetros da fluência da fala de discursos mentirosos. Método: Estudo quantitativo e transversal, com abordagem descritiva e analítica. Participaram da pesquisa vinte sujeitos distribuídos igualitariamente quanto ao gênero e escolaridade. Realizou-se uma filmagem da narrativa de dois discursos, um verdadeiro (pré-teste) e outro falso (teste), a partir da simulação de uma entrevista em que se utilizou máquina fotográfica no modo vídeo. Os dados foram tabulados em planilha Excel, comparados e submetidos às análises estatísticas pelo teste Qui-quadrado, pelo teste de distribuição normal e teste T, tendo como nível de significância $\mathrm{p}<$ 0,05. Resultados: A idade média dos participantes foi de 28,7 $\pm 9,8$. Os momentos pré-teste (verdade) e teste (mentira) não revelaram diferenças estatisticamente significantes quando a fluência foi analisada ou quando a quantidade de sílabas e palavras foi comparada. Houve correlação negativa (tanto na quantidade de sílabas quanto de palavras), indicando que quando um sujeito produz mais quantidade de palavras ou sílabas ao falar a verdade, tende a diminuir tal quantidade ao mentir. Conclusão: Os parâmetros da fluência na fala não possibilitaram distinguir os discursos verdadeiros dos mentirosos. Estudos com amostras maiores e que envolvam fatores estressores mais impactantes necessitam ser realizados para verificar se o estímulo teste foi ou não suficiente para alterar a fluência em discursos desonestos e assim, ratificar (ou não) os resultados deste estudo.
\end{abstract}

Palavras-chave: Detecção de mentiras; Fala; Fluência; Fonoaudiologia.

\begin{abstract}
Objectives: To analyze the speech fluency parameters of dishonest speak. Method: Quantitative and cross sectional study with descriptive and analytical approaches. Twenty subjects participated in the research equally distributed between gender and level of education. The narrative of two speeches was filmed, one true (pre-test) and the other false (test), from the simulation of an interview in which the camera is used in video mode. Data were tabulated in an Excel spreadsheet, compared and analyzed by statistical analysis using the chi-square test, the normal distribution test and the $t$ test, presenting significance level of $p<0,05$. Results: The mean age of participants was $28.7 \pm 9.8$. The pretest (truth) and test (lying) moments did not reveal statistically significant differences when fluency was analyzed or when the number of syllables and words was compared. There is negative correlation in both syllables and words quantities that indicates when a person is speaking the truth it has a higher frequency of words, or syllables, which lessen with a lie. Conclusion: The parameters of speech fluency did not make it possible to distinguish true speeches from liars. Studies with the largest and involving the most impacting stressors are carried out to verify whether or not
\end{abstract}


the test stimulus was sufficient to change the fluency in dishonest speeches and thus ratify (or not) the results of this study.

Keywords: Lie detection; Speech; Fluency; Speech, language and hearing sciences.

\begin{abstract}
Resumen
Objetivo: Analizar los parámetros de la fluidez del habla en los discursos mentirosos. Método: Estudio cuantitativo y transversal, con enfoque descriptivo y analítico. Veinte sujetos participaron en la investigación, igualmente distribuidos en términos de género y educación. Se filmó la narrativa de dos discursos, uno verdadero (pre-test) y el otro falso (test), a partir de la simulación de una entrevista en la que se utilizó una cámara en modo video. Los datos se tabularon en una hoja de cálculo de Excel, se compararon y se sometieron a análisis estadístico mediante la prueba de Chi-cuadrado, la prueba de distribución normal y la prueba $\mathrm{T}$, con un nivel de significancia de $\mathrm{p}<0.05$. Resultados: La edad media de los participantes fue $28,7 \pm 9,8$. Los momentos de prueba (verdad) y prueba (mentira) no revelaron diferencias estadísticamente significativas cuando se analizó la fluidez o cuando se comparó el número de sílabas y palabras. Hubo una correlación negativa (tanto en la cantidad de sílabas como de palabras), lo que indica que cuando un sujeto produce más palabras o sílabas al decir la verdad, tiende a reducir esta cantidad al mentir. Conclusión: Los parámetros de la fluidez del habla no permitieron distinguir los discursos verdaderos de los mentirosos. Se deben realizar estudios con muestras más grandes y que involucren factores estresantes más impactantes para verificar si el estímulo de la prueba fue o no suficiente para cambiar la fluidez en los discursos deshonestos y, por lo tanto, ratificar (o no) los resultados de este estudio.
\end{abstract}

Palabras clave: Detección de mentiras; Habla; Fluidez; Fonoaudiología.

\title{
1. Introdução
}

A Fonoaudiologia é a ciência que compreende os estudos, cuidados e desenvolvimento da comunicação humana, sendo que dentre os aspectos estudados e utilizados na prática profissional (no aperfeiçoamento e na reabilitação clínica) está a fluência, uma das áreas de atuação do fonoaudiólogo (Brasil, 2017; Crefono, 2021), que está relacionada à produção da fala. Dentre os aspectos analisados na fluência estão a continuidade, o ritmo, a entonação, a velocidade e/ou esforço com as quais as unidades fonológicas, lexicais, morfológicas e/ou sintáticas são produzidas, bem como as intenções comunicativas (Sassi, Campanati-Ortiz, \& Andrade, 2001).

Muitos fatores podem interferir em uma boa fluência da fala, dentre os quais, aventa-se a hipótese da emissão de discurso desonesto. O fenômeno da mentira caracteriza-se pela dissimulação de ideias, sentimentos e emoções (Matias, Leime, Bezerra, \& Torro-Alves, 2015), contudo, há poucos estudos brasileiros sobre o tema, evidenciando a importância de pesquisas na área (Brito, 2013; Matias et al., 2015).

A mentira pode ser detectada por expertises na área, que com o auxílio de treinamentos, conseguem analisar as incongruências entre o discurso e os recursos utilizados pelo falante (Brito, 2013). Quando não há fatos ou fundamentos extrínsecos para a detecção de mentiras, os examinadores precisam utilizar fatores intrínsecos dos indivíduos nas entrevistas (Curci, Lanciano, Battista, Guaragno, \& Ribatti, 2019). Dentre os sinais da mentira relacionados com a fluência encontra-se a mudança na velocidade da fala (Lobo, 2010), geralmente mais lentificada; o aumento de repetições (palavras ou sílabas) e de pausas (com o uso de pausas plenas); os erros de pronúncia e omissão de palavras (Pease \& Pease, 2005) tornando o discurso mais vago e repetitivo (Portella, 2006). Como tais argumentações são empíricas, ou seja, sem a análise da fluência propriamente dita, esta pesquisa foi planejada e desenvolvida.

Assim, o objetivo do presente estudo foi analisar os parâmetros da fluência da fala de discursos mentirosos e a quantidade de palavras e sílabas emitidas em discursos verdadeiros e desonestos.

\section{Metodologia}

A presente pesquisa foi aprovada pelo Comitê de Ética em Pesquisa (CAEE N 42025115.9.0000.5546), por ser um experimento que envolve a participação de seres humanos. Assim, os participantes assinaram o Termo de Consentimento Livre 
e Esclarecido e o Termo de Uso de Imagem e Voz. Trata-se de um estudo quantitativo (Pereira et al., 2018) e transversal, com abordagem descritiva e analítica.

A amostra foi constituída por vinte sujeitos ( $\mathrm{n}=10$ de cada gênero), com idade superior a dezenove anos, que foram convidados voluntariamente, no campus de uma universidade pública, a gravar discursos orais (verdadeiros e mentirosos) eliciados por narrativa desencadeada por perguntas de um entrevistador, simulando uma entrevista que seria gravada para análise da fluência. Para tanto, foi utilizada uma sala cedida pela instituição da pesquisa, composta por uma mesa - para o apoio de uma máquina fotográfica; uma cadeira disposta a um metro da máquina fotográfica e centralizada na frente da máquina; e outra cadeira para o instrutor sentar atrás da mesa e do equipamento, a fim de verificar a centralização da imagem do participante no equipamento e proferir as perguntas nos momentos pré-teste e teste em intensidade audível para compreensão do participante. A sala apresentava boa iluminação, ventilação e foram evitadas interrupções durante o procedimento.

Vinte e oito sujeitos foram convidados a participar, porém apenas vinte preencheram os critérios de elegibilidade. Como o objetivo era ter uma amostra igualitária entre os gêneros, a consulta para participação na pesquisa, inicialmente aleatória, foi tornando-se por conveniência para atender à demanda de igualdade de participantes de acordo com o gênero.

Foram incluídos na pesquisa sujeitos universitários com a faixa etária estipulada pela pesquisa, sem queixas e alterações na linguagem oral e audição. Foram excluídos sujeitos com queixas (pessoais e/ou familiares) e manifestações de disfluência gaga, outros distúrbios da comunicação, queixas de alterações neurológicas, psiquiátricas, que tenham sido submetidos a tratamento fonoaudiológico prévio ou que tenham declinado do convite para participar da pesquisa (timidez, não gostar de ser filmado, não querer elaborar um discurso falso, falta de tempo entre outros). Assim, antes da aplicação do procedimento, perguntas com os critérios de elegibilidade foram realizadas aos participantes.

O procedimento foi baseado em Pereira et al. (2006) e foi subdividido em dois momentos, sendo que no primeiro momento da entrevista (denominado por pré-teste) o participante respondia às perguntas do entrevistador falando a verdade a fim de poder ser analisada a linha de base e os recursos utilizados na produção da sua fala (com perguntas sobre: idade, curso em que está matriculado/com que trabalha e o motivo de escolha desse curso/trabalho). Esse momento é importante para poder ser composta a linha de base do sujeito, que nesta pesquisa, compreendeu em analisar as características da fluência dos participantes. Em um segundo momento, houve a solicitação da produção de um discurso desonesto/mentiroso (situação teste), para posterior comparação com o momento pré-teste (linha de base). Para tanto, perguntas como dizer o nome dos pais, irmãos, bairro onde mora, o nome de uma pessoa que gosta e o motivo de gostar dessa pessoa foram efetivadas. O tempo de gravação foi de, no máximo, cinco minutos.

O entrevistador foi previamente treinado para a aplicação da entrevista e ajustes no equipamento, apresentando um script para seguir o procedimento da mesma forma com todos os participantes, sendo que um mesmo entrevistador aplicou a proposta em todos os participantes.

O discurso oral foi literalmente transcrito de acordo com Andrade (2004), considerando como disfluência comum a presença de hesitações, interjeições, revisões, palavras não terminadas, repetição de palavras, de segmentos e de frases e como disfluência gaga, a presença de bloqueios, prolongamentos, pausas iguais ou maiores do que dois segundos, presença de repetições de fonemas, de sílabas e intrusão de sons ou segmentos (C. R. F. de Andrade, 2004, 2012).

Outra análise foi realizada, qual seja, a da quantidade de palavras e sílabas expressas no tempo de um minuto, nos momentos pré-teste (discurso que correspondia à verdade) e teste (produção de discurso mentiroso). Não foi considerado um parâmetro de normalidade ou de alteração, tendo em vista que a análise foi realizada pela comparação da quantidade de palavras e sílabas interindivíduo nos dois momentos do teste, bem como não foi possível a obtenção mínima de 200 sílabas expressas, como recomendado pela literatura (C. R. F. de Andrade, 2004). 
Todas as produções de fala foram registradas em máquina fotográfica na função de vídeo, com uso da máquina fotográfica Sony Cyber Shot, digital (7,2 mega pixels), modelo DSC P200. Para tanto, a máquina foi colocada sobre um tripé (para evitar alterações decorrentes de má postura do fotógrafo), ajustada a uma altura de um metro do solo e à distância de dois metros de um fundo constante.

Os resultados foram analisados pelo Teste Qui-quadrado comparando-se a fluência nos momentos pré-teste (discurso verdadeiro) e teste (discurso falso), adotando-se relevância de 5\%, pelo teste T e Pearson para verificar se a quantidade de sílabas e palavras se modificaram nos momentos pré-teste e teste.

\section{Resultados}

A média de idade dos participantes foi 28,7 anos (desvio padrão=9,8; mínimo=19; máximo=57). Houve distribuição igualitária entre os gêneros (50\% masculino e 50\% feminino), apresentando nível de escolaridade superior incompleto.

Na Tabela 1 encontram-se os resultados obtidos a partir da análise da fluência nos momentos pré-teste e teste dos dois grupos participantes da investigação.

Tabela 1. Análise da fluência nas situações pré-teste e teste dos participantes da pesquisa.

\begin{tabular}{|c|c|c|c|c|c|c|c|}
\hline \multicolumn{4}{|c|}{ Pré-Teste (Verdade) } & \multicolumn{4}{|c|}{ Teste (Mentira) } \\
\hline \multicolumn{8}{|c|}{ Com alterações da } \\
\hline \multicolumn{2}{|c|}{ Sem alterações na fluência } & \multicolumn{2}{|c|}{ fluência } & \multicolumn{2}{|c|}{ Sem alterações da fluência } & \multicolumn{2}{|c|}{ Com alterações da fluência } \\
\hline $\mathbf{N}$ & $\%$ & $\mathbf{N}$ & $\%$ & $\mathbf{N}$ & $\%$ & $\mathbf{N}$ & $\%$ \\
\hline 19 & 95 & $1 *$ & 5 & 15 & 75 & $5 * *$ & 25 \\
\hline
\end{tabular}

* presença de hesitação. ** presença de pausas superiores a dois segundos, hesitação, interjeições e intrusões de palavras. Fonte: Autores.

Das alterações da fluência no momento do teste, três foram relacionados às pausas, como pode ser observado na Tabela 2.

Tabela 2. Análise das pausas nas situações pré-teste e teste realizadas pelos participantes da pesquisa.

\begin{tabular}{|c|c|c|c|c|c|c|c|}
\hline \multicolumn{4}{|c|}{ Pré-Teste (Verdade) } & \multicolumn{4}{|c|}{ Teste (Mentira) } \\
\hline \multicolumn{2}{|c|}{ Sem alterações } & \multicolumn{2}{|c|}{ Com alterações } & \multicolumn{2}{|c|}{ Sem alterações } & \multicolumn{2}{|c|}{ Com alterações } \\
\hline $\mathbf{N}$ & $\%$ & $\mathbf{N}$ & $\%$ & $\mathbf{N}$ & $\%$ & $\mathbf{N}$ & $\%$ \\
\hline 20 & 100 & 0 & 0 & 17 & 85 & 3 & 15 \\
\hline
\end{tabular}

Qui-quadrado $p=1,00$

Fonte: Autores.

Para verificar se havia distribuição normal da amostra entre palavras e sílabas, foi feito o teste de distribuição normal, sendo constatados gráficos, para ambas situações, de normalidade. Na Tabela 3 encontram-se os resultados obtidos a partir da análise da quantidade de sílabas e palavras nos momentos pré-teste e teste dos participantes da investigação.

Tabela 3. Análise da quantidade de sílabas e palavras nos momentos pré-teste e teste produzidas pelos participantes da 
pesquisa.

\begin{tabular}{llllll}
\hline \multirow{2}{*}{ Quantidade Média de } & \multicolumn{2}{l}{ Pré-Teste (Verdade) } & \multicolumn{2}{l}{ Teste (Mentira) } & Teste T \\
\cline { 2 - 6 } & N & DP & N & DP & 0,83 \\
Sílabas & 55,65 & $\pm 32,43$ & 57,90 & $\pm 29,04$ & 0,99 \\
Palavras & 26,55 & $\pm 15,94$ & 26,50 & $\pm 10,95$ & \\
\hline
\end{tabular}

Fonte: Autores.

\section{Discussão}

Para que a fala seja fluente faz-se necessária uma transmissão suave, contínua e sem esforço dos sons da fala (C. R. F. de Andrade, 2000, 2004; C. R. F. de Andrade, Cervone, \& Sassi, 2003; Barbosa, 2005), com harmonia entre a velocidade de fala, os níveis linguísticos do falante e sua capacidade motora orofacial (Merlo, 2007), sendo sua produção de ordem complexa, tendo em vista que exige um conjunto de órgãos e sistemas trabalhando sincronicamente (Ventura et al., 2010).

Diversos fatores podem influenciar a fluência, como a idade que sofre influência direta na velocidade de fala, com a aquisição e desenvolvimento dessa habilidade, estabilização para posterior degeneração do padrão de velocidade no decorrer da vida (Martins \& Andrade, 2008); a hereditariedade; a integridade do sistema nervoso central (Andrade, 2000; Merlo, 2007); a cognição; as demandas sociais; as capacidades linguísticas, práxicas verbais e perceptuais auditivas (Andrade, 2000); a memória (Andrade, 2000; Rodrigues, Yamashita, \& Chiappetta, 2008); os estigmas sociais, como a percepção do sujeito enquanto um ser falante; o estado emocional (Friedman, 1999); a ansiedade (Toazza et al., 2016); a produção de discursos mentirosos (Lobo, 2010; Pease \& Pease, 2005), entre outros.

Se um sujeito que não apresenta disfluência gaga em situações cotidianas, ao mentir essa habilidade poderia ser modificada? Ou ainda, pode haver aumento da disfluência comum em discursos desonestos? Se a resposta for afirmativa para ambas perguntas, então a capacidade de identificar um discurso mentiroso pela análise da fluência poderia facilitar sua detecção e foi partindo dessa questão de pesquisa que este estudo foi desenvolvido.

Desta forma, foi eleita a estratégia de entrevista com os participantes, tendo em vista que esta atividade poderia produzir um aumento artificial de dificuldade cognitiva, o que poderia favorecer a presença de sinais reveladores de sobrecarga cognitiva (Blandón-Gitlin, López, Masip, \& Fenn, 2017).

Além disso, ao ser realizado o momento pré-teste, em que perguntas impessoais e gerais foram realizadas, pode-se facilitar a familiarização do participante com o procedimento, sendo tais perguntas favorecedoras de discursos verdadeiros (Masip \& Herrero, 2015) e, no teste, ao se solicitar perguntas de cunho emocional associado ao ato de mentir, pode favorecer o desencadeamento da ansiedade presumida (Vasconcellos et al., 2016) e assim, sinais de sobrecarga cognitiva (Masip \& Herrero, 2015) que poderiam prejudicar a fluência verbal. No entanto, nossos dados resultaram em inexistência de diferenças contrastivas que pudessem diferenciar os depoimentos verdadeiros dos falsos, sendo levantadas algumas hipóteses para tais resultados.

A primeira, de que a entrevista proposta não foi um fator suficientemente forte para gerar algumas das características citadas por alguns autores (Lobo, 2010; Pease \& Pease, 2005). Para a mentira se manifestar é necessário que o entrevistador adote uma postura ativa, manejando estrategicamente a interação (Masip \& Herrero, 2015). No caso da presente pesquisa, foi seguido um procedimento padrão para que não houvesse viés de resposta e talvez este procedimento tenha interferido nos resultados obtidos.

A segunda estaria relacionada ao número amostral, pequeno e, talvez, insuficiente para fazer uma generalização. Um estudo argumentou que o tamanho da amostra é de fundamental relevância na área de saúde, em virtude das particularidades da área, já que pesquisa a complexidade do ser humano (Weyne, 2004). Portanto, esta é uma das limitações deste estudo. 
A terceira e última hipótese, de que a fluência não se altera em situações de discursos mentirosos, como evidenciando no grupo de estudo investigado, independentemente da carga estressora.

No entanto, cabe ressaltar que apesar de não haver diferenças estatisticamente significativas intersujeitos nos momentos pré-teste e teste, houve um ligeiro aumento da disfluência (três sujeitos que não apresentaram alterações nas pausas, as apresentaram), ratificando o exposto por outros autores (Pease \& Pease, 2005). A presença de pausas, hesitações e a intrusão de palavras e interjeições podem tornar um discurso mais lento. Nesta pesquisa, um sujeito manteve a hesitação nos momentos pré-teste e teste e outro sujeito apresentou aumento de interjeições e intrusões de palavras (como ãh, aí, é, então). Lobo (2010) comentou a possibilidade de uma fala mais lentificada, porém não encontramos repetições de sílabas ou palavras, bem como erros de pronúncia e omissão de palavras como anunciado por Pease \& Pease (2005), nem tampouco um discurso mais vago e repetitivo, como afirmado por Portella (2006).

No campo da detecção da mentira, uma revisão evidenciou ferramentas recentes de detecção de mentira baseadas na teoria cognitiva (Walczyk, Sewell \& DiBenedetto, 2019) e um outro estudo apresentou a possibilidade de detecção por meio do rastreamento ocular em circunstâncias laboratoriais (Ge et al., 2020). Além disso, existem três métodos de base distintos e importantes desenvolvidos com a intenção de elevar a precisão na detecção: métodos na medição de alterações fisiológicas, os de análise do conteúdo da fala e os de observação do comportamento (Vrij, 2000).

Os métodos de medição de alterações fisiológicas, contam com aparelhos como eletroencefalograma (EEG), ressonância magnética funcional (RMIf) e polígrafo, que não são uteis para uso jurídico no Brasil, dado impedimento legal (Vrij, Edward, \& Bull, 2001).

Os métodos de análise do conteúdo da fala incluem a técnica de análise de conteúdo baseada em critério (ACBC) e a técnica de monitoramento da realidade (MR). A primeira técnica busca distinguir um relato falso de um verdadeiro por um meio de verificação da fonte de informação (se o indivíduo vivenciou um fato ou o inventou), utilizando-se de testes psicométricos, entrevistas semiestruturadas, técnicas de análise sistemáticas e julgamento clínico (Kohnken, 2004). A segunda técnica - MR - tem objetivo de verificar se as memórias que um indivíduo relata derivam de uma experiência interna ou externa, sendo que as externas contêm mais informações (sensorial, contextual, afetiva e semântica) e as memórias internas tem mais referências a operações cognitivas (Siegried, 2004).

Há ainda a divisão dos métodos de detecção da mentira em objetivos e subjetivos. Os objetivos visam direcionar atenção para a frequência de emissão de alguns sinais não verbais que indicam mentiras (tempo de fala, latência da resposta, pausas e movimentos de mão). Entretanto, essa técnica requer a gravação em vídeo e posterior análise do comportamento do suspeito. Um exemplo deste tipo de técnica é o sistema de codificação de ação facial (FACS), desenvolvido por Ekman \& Friesen (1974). Utilizando esse sistema, é possível inferir a emoção que está provocando uma determinada expressão facial e assim, pode-se inferir se uma pessoa está mentindo ou dizendo a verdade no seu relato verbal. Já os subjetivos são menos dispendiosos e subdividem-se em dois: o direto e o indireto. No método de deteç̧ão direta ou explícita, o participante é solicitado apenas para julgar se outra pessoa está mentindo ou não e a precisão não difere significativamente da precisão ao acaso e no indireto, o participante é solicitado a prestar atenção em certas características gerais do comportamento alheio (ambivalência, confiança ou esforço para pensar), permitindo a deteç̧ão de mentiras com maior precisão (De Paulo \& Morris, 2004).

Frente ao exposto, a tentativa desta pesquisa foi o de propor uma avaliação, o mais objetiva possível, já que autores que comentaram sobre a possível alteração da fluência no ato da mentira (Lobo, 2010; Pease \& Pease, 2005) não propuseram investigações próprias para tal propósito. No entanto, mudanças metodológicas (com perguntas mais abertas, maior número de sujeitos participantes e perguntas com maior carga de estresse comunicativo) serão necessárias para ratificar ou não os dados obtidos neste estudo piloto. 
Além disso, sugere-se a idealização de um protocolo para a análise da fluência em discursos verdadeiros e falsos com perguntas mais abertas a fim de que sejam feitas análises de velocidade de fala padronizadas, bem como enviar as amostras de áudio para especialistas em disfluência para verificar se tais profissionais conseguem distinguir os discursos honestos dos mentirosos, sem identificar que momento do teste é ou não verdadeiro.

No Brasil foi encontrado um número reduzido de estudos sobre a mentira, requerendo uma equipe multidisciplinar na organização e planejamento de estudos mais completos na área, incluindo a Fonoaudiologia (Quinta \& Coelho, 2009). Há um estudo recente a respeito da deteç̧ão da mentira por meio da voz, conduzido por uma equipe multidisciplinar com maioria composta por profissionais fonoaudiólogos, que identificou diferenças em aspectos vocais, como a tensão e o ataque vocal entre os discursos honestos e falsos (Santos et al., 2021), inferindo-se a necessidade de maior objetividade nos estudos da área.

\section{Conclusão}

Os parâmetros da fluência na fala nos momentos pré-teste (discurso verdadeiro) e teste (discurso desonesto) não evidenciaram diferenças estatisticamente significativas, embora um ligeiro aumento de pausas, hesitações, interjeições e intrusão de palavras tenha sido observado no momento teste. Desta forma, a hipótese inicial de que a emissão de discursos desonestos pode alterar a fluência da fala foi rejeitada, suscitando a necessidade de mais estudos na área.

Sugere-se a realização de estudos com amostras maiores e que envolvam fatores estressores mais impactantes necessitam ser realizados, a fim de verificar se o estímulo teste foi ou não suficiente para alterar a fluência em discursos mentirosos/desonestos e assim, ratificar (ou não) os resultados deste estudo piloto.

\section{Referências}

Andrade, C. R. F. de (2000). Processamento da fala: aspectos da fluência. Pró Fono, 12, 69-71.

Andrade, C. R. F. de (2004). Fluência. In C. Andrade \& D. Befi-Lopes (Eds.), ABFW: teste de linguagem infantil nas áreas de fonologia, vocabulário, fluência e pragmática. (2nd ed.). Pró-Fono.

Andrade, C. R. F. de (2012). Protocolos. In CRF de Andrade (Ed.), Gagueira infantil: risco, diagnóstico e programas terapêuticos. Pró-Fono.

Andrade, C. R. F. de, Cervone, L. M., \& Sassi, F. C. (2003). Relationship between the stuttering severity index and speech rate. Sao Paulo Medical Journal, 121(2), 81-84. 10.1590/S1516-31802003000200010

Barbosa, L. M. G. (2005). Noções básicas sobre a gagueira: suas características, sua etiologia e as teorias sobre sua natureza. In I. Marchesan \& J. Zorzi (Eds.), Conhecimentos essenciais para atender bem a pessoa com gagueira (2nd ed.),Pulso.

Blandón-Gitlin, I., López, R.M., Masip, J., \& Fenn, E. (2017). Cognición, emoción y mentira: implicaciones para detectar el engaño. Anuario de Psicología Jurídica, 27(1), 95-106. 10.1016/j.apj.2017.02.004

Brasil, C. F. de F. Resolução do Conselho Federal de Fonoaudiologia no 507. Pub. L. nº 507 (2017). Brasil.

Brito, T. D. de Q. (2013). O efeito do treino na detecção direta da mentira. Universidade de Brasília.

Crefono. (2021). História da Fonoaudiologia: o que é Fonoaudiologia? http://www.crefono4.org.br/institucional/historia

Curci, A., Lanciano, T., Battista, F., Guaragno, S., \& Ribatti, R. M. (2019). Accuracy, Confidence, and Experiential Criteria for Lie Detection Through a Videotaped Interview. Frontiers in Psychiatry, 9. https://doi.org/10.3389/fpsyt.2018.00748

De Paulo, B., \& Morris, W. (2004). Cues to deception and indirect lie detection. In P. A. Granhag \& L. Stromwall (Eds.), The detection of deception in forensin (pp. 15-40). Cambridge University Press.

Ekman, P., \& Friesen, W. (1974). Unmasking the face: a guide to recognizing emotions from facial expressions. Consulting Psychology Press Inc.

Friedman, S. (1999). Fluência: normalidade e patologia. Distúrbios Da Comunicação, 11, 131-136.

Ge, F. F., Yang, X. Q., Chen, Y. X., Huang, H. L., Shen, X. C., Li, Y., \& Hu, J. M. (2020). Application of Eye Tracker in Lie Detection. Fa Yi Xue Za Zhi, 36(2), 229-232. https://doi.org/10.12116/j.issn.1004-5619.2020.02.015

Kohnken, G. (2004). Statement validity. In P. Granhag \& L. Stromwall (Eds.), The detection of deception in forensin (pp. 64-102). Cambridge University Press. 
Research, Society and Development, v. 10, n. 10, e534101019114, 2021

(CC BY 4.0) | ISSN 2525-3409 | DOI: http://dx.doi.org/10.33448/rsd-v10i10.19114

Lobo, M. (2010). Por que as pessoas mentem? Arte Editorial.

Martins, V. de O., \& Andrade, C. R. F. de (2008). Perfil evolutivo da fluência da fala de falantes do português brasileiro. Pró-Fono Revista de Atualização Científica, 20(1), 7-12. 10.1590/S0104-56872008000100002

Masip, J., \& Herrero, C. (2015). New approaches in deception detection II. Active interviewing strategies and contextual information. Papeles Del Psicologo, 36(2), 96-108.

Matias, D. W. de S., Leime, J. L., Bezerra, C. W. A. G., \& Torro-Alves, N. (2015). Mentira: Aspectos Sociais e Neurobiológicos. Psicologia: Teoria e Pesquisa, 31(3), 397-401. 10.1590/0102-37722015032213397401

Merlo, S. (2007). Algumas reflexões sobre o conceito de fluência. In E. Rocha (Ed.), Gagueira: um distúrbio de fluência (pp. 55-79). Santos.

Pease, A., \& Pease, B. (2005). Desvendando os segredos da linguagem corporal. Sextante.

Pereira, A., Shitsuka, D., Parreira, F., \& Shitsuka, R. (2018). Metodologia da pesquisa científica. Núcleo de Tecnologia Educacional da Universidade Federal de Santa Maria

Pereira, M. E., Brasileiro, R., Silva, J. F. da, Silva, P. B. e, Brachi, D., \& Albuquerque, F. (2006). Estereótipos, mentiras e videotape: estudos experimentais sobre a acurácia na identificação da mentira. Psicologia Em Estudo, 11(1), 209-218. 10.1590/S1413-73722006000100024

Portella, M. (2006). Como identificar a mentira: sinais não verbais da dissimulação. QualityMark.

Quinta, N.C. de C., \& Coelho, C. (2009). Contando e detectando mentiras: efeito do feedback sobre o desempenho. Psicologia: Teoria e Pesquisa, 25(1), 137145. 10.1590/S0102-37722009000100016

Rodrigues, A. B., Yamashita, É. T., \& Chiappetta, A. L. de M. L. (2008). Teste de fluência verbal no adulto e no idoso: verificação da aprendizagem verbal. Revista CEFAC, 10(4), 443-451. 10.1590/S1516-18462008000400004

Santos, N. M., Bispo, J. A. R., Santos, M. de J., Santos, J. M. de J., Pellicani, A. D., \& César, C. P. H. A. R. (2021). Discursos honestos e mentirosos: Há como discriminá-los pela voz? Research, Society and Development, 10(9), e46610918266. 10.33448/rsd-v10i9.18266

Sassi, F., Campanati-Ortiz, H., \& Andrade, C. (2001). Terminologia: fluência e desordens da fluência. Pró Fono, 13, 107-113.

Siegried, S. (2004). Reality monitoring and the detection of deception. In The detection of deception in forensin (pp. 41-63). Cambridge University Press.

Toazza, R., Salum, G. A., Jarros, R. B., Sousa, D., Salles, J. F. de, \& Manfro, G. G. (2016). Phonemic verbal fluency and severity of anxiety disorders in young children. Trends in Psychiatry and Psychotherapy, 38(2), 100-104. 10.1590/2237-6089-2016-0018

Vasconcellos, S. J. L., Santos, B. S. dos, Morais, L. Q., Ferraz, R. C., Freitas, P. O. de, \& Silveira, J. F. (2016). Evidências de validade de uma escala para ansiedade em situações de mentira. Revista Avaliação Psicológica, 15(3), 383-390. 10.15689/ap.2016.1503.11.John Wiley and Sons Ltd.

Vrij, A., Edward, K., \& Bull, R. (2001). Police officers hability to detect deceit: the benefit of indirect deception detection measures. Legal and Criminological Psychology, 6, 185-196.

Walczyk, J. J., Sewell, N., \& DiBenedetto, M. B. (2018). A Review of Approaches to Detecting Malingering in Forensic Contexts and Promising Cognitive Load-Inducing Lie Detection Techniques. Frontiers in Psychiatry, 9. https://doi.org/10.3389/fpsyt.2018.00700

Weyne, G. (2004). Determinação do tamanho da amostra em pesquisas experimentais na área de saúde. Arquivos Médicos do ABC, 29, 87-90. 